\title{
Improvement of cardiac functions after renal transplant in pediatric patients with severe cardiac risk
}

\author{
Esra Baskin ${ }^{1}$, Kaan Gulleroglu ${ }^{1}$, llkay Erdogan² ${ }^{2}$ Birgul Varan² ${ }^{2}$ Aydincan Akdur ${ }^{3}$, Gokhan Moray $^{3}$, Mehmet Haberal $^{3}$ \\ ${ }^{1}$ Department of Pediatric Nephrology, Baskent University Hospital, Ankara, Turkey \\ ${ }^{2}$ Department of Pediatric Cardiology, Baskent University Hospital, Ankara, Turkey \\ ${ }^{3}$ Department of General Surgery, Baskent University Hospital, Ankara, Turkey
}

Background: Patients with chronic kidney disease (CKD) are at increased risk for cardiovascular morbidity and mortality. The surgical procedure is risky in these patients, but renal transplant (RTX) reduces cardiac mortality and the risk for development of chronic cardiac insufficiency compared with long-term dialysis. We presented patients with high cardiovascular risk and the success of RTX on the cardiac functions.

Methods: Since 1975, our center has performed 3,109 kidney transplant procedures, with 360 involving pediatric patients. We retrospectively evaluated data from the past 10 years for 165 pediatric patients with RTX. Cardiac functions of the patients were evaluated in detail before and after RTX. Patients with severe cardiac risk were identified, and the effects of successful RTX on cardiac functions were investigated.

Results: Severe cardiac risk was detected in 11 patients (six females and five males). Mean age of patients at transplant was $12.48 \pm 3.60$ years, and mean follow-up period was $4.16 \pm 1.34$ years after transplant. Preoperative mean ejection fraction significantly increased after RTX within 6 months $(37.45 \% \pm 9.77 \%$ and $66.45 \% \pm 8.39 \%$, respectively; $P<0.01)$ and mean left ventricle diastolic and systolic diameter were significantly decreased, after RTX within 6 months $(52.58 \pm 8.58 \mathrm{~mm}$ vs. $42.86 \pm 9.25 \mathrm{~mm}$ and $42.57 \pm 8.16 \mathrm{~mm}$ vs. $27.05 \pm 8.24 \mathrm{~mm}$, respectively; $\mathrm{P}<0.01$ ). This significant improvement on cardiac functions persisted 2 years after RTX.

Conclusions: After RTX, cardiac functions improved markedly in patients with end-stage renal disease and severe cardiac risk. We suggest that although the surgical procedure is risky, RTX should be considered the treatment of choice for these patients, because a longer duration of dialysis in these patients may result in progressive and ultimately irreversible myocardial dysfunction.

Corresponding author: Esra Baskin

E-mail: rectorate@baskent.edu.tr

(C) The Korean Society for Transplantation

This is an Open Access article distributed under the terms of the Creative Commons Attribution Non-Commercial License (http://creativecommons.org/licenses/by-nc/4.0/) which permits unrestricted non-commercial use, distribution, and reproduction in any medium, provided the original work is properly cited. 